\title{
Sequelae and management of radiation vasculopathy in neurosurgical patients
}

\author{
Spencer Twitchell, BS, Michael Karsy, MD, PhD, Jian Guan, MD, William T. Couldwell, MD, PhD, \\ and Philipp Taussky, MD
}

\begin{abstract}
Department of Neurosurgery, Clinical Neurosciences Center, University of Utah, Salt Lake City, Utah
The term "radiation vasculopathy" defines a heterogeneous and poorly defined complex of vessel injury due to radiation. Radiation vasculopathy remains underrecognized and poorly treated with respect to head and neck radiotherapy. Distinct injury patterns to small $(\leq 100-\mu \mathrm{m})$, medium $(>100-\mu \mathrm{m})$, and large $(>500-\mu \mathrm{m})$ vessels can occur, resulting in carotid stenosis, intracranial stenosis, and vascular anomalies (e.g., cavernous malformations, aneurysms). Because of the lack of clinical evidence and guidelines, treatment plans involve medical management, carotid endarterectomy, and carotid artery stenting and are developed on a patient-by-patient basis. In this review, the authors discuss the current pathophysiology, imaging, clinical impact, and potential treatment strategies of radiation vasculopathy with clinical pertinence to practicing neurosurgeons and neurologists. A review of 4 patients with prior head and neck tumors in whom delayed radiation vasculopathy developed after radiotherapy demonstrates the application of various treatment options in a caseby-case manner. Earlier recognition of radiation vasculopathy disease patterns may enable earlier initiation of treatment and monitoring for complications. Standardized terminology and treatments may assist with improving clinical outcomes. https://thejns.org/doi/abs/10.3171/2017.12.JNS172635
\end{abstract}

KEYWORDS radiation vasculopathy; vasculitis; management; aneurysm; carotid stenosis; vascular disorders

$\mathrm{R}$ ADIATION vasculopathy is defined as the complex pathological reorganization of vascular tissue after radiation exposure. The initial description of radiation vasculopathy ${ }^{11}$ has been further elucidated by more recent studies implicating the direct impact of radiation on vessel injury. ${ }^{10,22}$ Acute and chronic injury occurs after head and neck radiotherapy, which can result in microvascular thrombosis, acute vascular rupture, and accelerated atherosclerotic disease of medium arteries $>$ $100 \mu \mathrm{m}$ ), as well as injury to the vasa vasorum and occlusive vasculopathy of large arteries $(>500 \mu \mathrm{m}) .{ }^{8}$ Radiation vasculopathy includes carotid stenosis, intracranial vessel stenosis, vasculitis, and cerebral ischemia. Dysplastic vascular organization, such as moyamoya patterns of transdural vessel anastomoses, and vascular abnormalities, such as aneurysms and cavernous malformations, are also observed. Radiation-induced aneurysms, resulting from injury of endothelium that promotes aneurysm formation, can demonstrate a high rate of rupture and adjacent vessel stenosis. ${ }^{17,21}$ Microvascular injury can also result in delayed cognitive impairment, ${ }^{16}$ cranial nerve injury, ototoxicity, and endocrinopathy? ${ }^{7}$ Stroke-like migraine attacks after radiation therapy (SMART) syndrome is a rare, poor- ly defined entity with migraine-like headaches and focal neurological signs and seizures lasting days to weeks associated with vascular reactivity. ${ }^{15}$ Although vessel rupture and other acute injuries are less common findings with modern treatment, occlusive vasculopathies are now being seen years after initial radiotherapy with an increased incidence because of improved overall oncological treatments and patient survival.

Despite the impact of radiation vasculopathy, it is often underrecognized and poorly managed. Incidences of $18 \%-40 \%$ for significant carotid stenosis ${ }^{2,5,23,25}$ and $12 \%-$ $21 \%$ for stroke at 20 years ${ }^{3,9}$ have been reported after head and neck radiotherapy. Larger case-control and cohort studies have suggested at least a doubled risk ratio for transient ischemic attack and stroke after head and neck radiotherapy. ${ }^{2,4}$ Haddy et al. ${ }^{13}$ evaluated 4227 patients who underwent radiotherapy for childhood cancer and survived at least 5 years. With a mean follow-up of 29 years, 23 deaths from cerebrovascular diseases were identified. Although there was significant heterogeneity of doses and treatment durations, radiation treatment to the prepontine cistern resulted in the highest risk of injury (17.8-fold difference for patients with $>50-$ Gy treatment compared with $<0.1 \mathrm{~Gy}$ ),

ABBREVIATIONS $\mathrm{ACOA}=$ anterior communicating artery; $\mathrm{CAS}=$ carotid artery stenting; $\mathrm{CEA}=$ carotid endarterectomy; $\mathrm{DSA}=$ digital subtraction angiography; $\mathrm{ICA}=$ inter nal carotid artery; MRA = MR angiography; PED = Pipeline embolization device.

SUBMITTED October 20, 2017. ACCEPTED December 19, 2017.

INCLUDE WHEN CITING Published online June 1, 2018; DOI: 10.3171/2017.12.JNS172635. 
and the relationship between dose and risk of death from cerebrovascular cause was linear. Additional risk factors include radiation during childhood, genetic risk factors (e.g., neurofibromatosis type 1), concomitant chemotherapy (e.g., cisplatin), and radiation fields, including the supraclinoid carotid, circle of Willis, or posterior fossa.

Significant heterogeneity in tumor types and radiation protocols limit comparability of studies and management strategies. Medical management, including anticoagulation and antilipid therapies, remains poorly studied in radiation vasculopathy. Recent advances in endovascular carotid artery stenting (CAS) have supplemented prior approaches in carotid endarterectomy (CEA) and vessel bypass. Because of the widespread expansion of radiotherapy approaches and longer patient survival, increased recognition of radiation vasculopathy by neurosurgeons and neurologists is critical to initiate early treatment strategies when warranted. This focused review will discuss some of the modern approaches to the management of radiation vasculopathy; we also present 4 case examples.

\section{Pathophysiology}

Recent studies have elucidated the complex formation of atherosclerotic plaque and the impact of radiation on this process. ${ }^{1,12,19}$ Atherosclerosis of medium-sized vessels involves a combination of lipoprotein accumulation, inflammation, and distinct involvement of endothelial, smooth muscle, and immune cells. ${ }^{26}$ Radiation can induce early cell apoptosis within hours of treatment via a variety of mechanisms, including increased cell-cycle checkpoint proteins and p53 expression; increased acid sphingomyelinase activity; increased secretion of cytokines, such as tumor necrosis factor- $\alpha$ and transforming growth factor$\beta 1$; and upregulated transcription factors, including cyclic adenosine monophosphate (cAMP) and cAMP response element-binding protein. Injury to the neural progenitor cells located in the subventricular zone and hippocampus is thought to mediate the delayed cognitive impairment seen after radiation therapy with stable follow-up imaging by preventing full cortical neurogenesis and pruning. ${ }^{16}$ Increased oxidative stress from reactive oxygen species (ROS) activates microglia. ROS formation also disrupts endothelial cells, resulting in apoptosis, adventitial fibrosis, vessel wall and basement membrane thickening, vessel dilation, and increased permeability from loss of tight junctions. Newly formed blood vessels are leaky and lack pericytes and smooth muscle cell-supporting structures, which makes the vessels more unstable. Vessels undergo hyalinization and fibrinoid necrosis, along with endothelial proliferation and perivascular inflammatory infiltration.

Preclinical approaches toward ameliorating radiation vasculopathy have involved reduction of postradiation inflammation and blocking of related downstream pathways, use of neuroprotective agents (e.g., erythropoietin), transplantation of neural stem cells, use of angiogenesis inhibitors (e.g., bevacizumab), treatment with hyperbaric oxygen, and blockade of the renin-angiotensin system pathway. Regardless, the application of these strategies clinically remains limited.

\section{Imaging Findings}

Imaging findings of radiation vasculopathy show a predictable location and time course. ${ }^{27}$ Acute injury occurs within several weeks of treatment, early delayed injury within several weeks to months, and late injury months to years after treatment. Acute injury involves capillary permeability and vasodilation appearing as vasogenic edema. Current protocols to treat patients undergoing radiotherapy with corticosteroids are aimed at reducing acute injury. Early delayed injury involves both vasogenic edema and demyelination, whereas late injury involves white matter necrosis, focal or diffuse demyelination, astrocytosis, cerebral atrophy, and necrotizing leukoencephalopathy. Vessel changes can be focal or diffuse, depending on radiation fields and dose, and white matter shows the most sensitivity to radiation treatments. CT imaging findings show white matter hypodensity, vasogenic edema, and irregular vascular patterns with contrast administration. Similarly, MRI and MR angiography (MRA) reflect these findings, showing white matter T2 signal hyperintensity, hypointense lesions suggestive of vascular anomalies (e.g., cavernous malformations or telangiectasias), diffuse leukodystrophy, gliosis, and demyelination. Digital subtraction angiography (DSA) findings show moyamoya revascularization patterns and multifocal vessel narrowing. Vessel imaging studies can help to identify radiation-induced aneurysms.

\section{Tissue Radiosensitivity}

The dose, duration, and tissue type during radiotherapy play important roles in the formation of radiation vasculopathy. ${ }^{7}$ The latency of radiation vasculopathy diagnosis ranges from 2 to 25 years, with a peak incidence at 3 years. ${ }^{10,20,27}$ Optic neuropathy occurs $6-24$ months after treatment with 8-10 Gy for stereotactic fields, while mild transient injury can be seen with sellar treatment protocols (45-55 Gy). ${ }^{14}$ Ototoxicity, endocrinopathy (e.g., growth hormone [50\%], gonadotropin [25\%], hyperprolactinemia [24\%], adrenocorticotropic hormone [19\%], and primary hypothyroidism [16\%]), and secondary tumor formation (e.g., meningiomas, malignant gliomas, nerve sheath tumors, and sarcomas) occur in a dose-dependent manner.

\section{Treatment of Radiation Vasculopathy}

Whereas medical treatment and primary prevention of stroke are well studied in atherosclerotic and thromboembolic mechanisms, the medical management of radiation vasculopathy remains unclear. ${ }^{6}$ Beyond treatment of classic cardiovascular risk factors (e.g., hypertension, diabetes, atrial fibrillation, tobacco use), preventative treatment with anticoagulation or antilipid medications specifically for radiation vasculopathy has not been studied extensively. In addition, patients treated for radiation vasculopathy likely have hypercoagulability due to their underlying cancer history. Several recent randomized clinical trials have supported the use of anticoagulation for patients with cancer and deep venous thrombosis, showing improved survival among these patients. However, similar trials for primary stroke prevention remain outstanding. Medical treatment of radiation vasculopathy remains a patient-by- 
TABLE 1. Summary of pertinent case information

\begin{tabular}{|c|c|c|c|c|c|}
\hline $\begin{array}{l}\text { Case } \\
\text { No. }\end{array}$ & $\begin{array}{l}\text { Age }(y r s) \\
\text { Sex }\end{array}$ & $\begin{array}{l}\text { FU Length } \\
\text { (mos) }\end{array}$ & Tumor Type & Treatment & Diagnostic Imaging \\
\hline 1 & $37, \mathrm{M}$ & 23.9 & Rt frontal astrocytoma & $\begin{array}{l}\text { Pterional craniotomy \& aneurysm } \\
\text { trapping }\end{array}$ & $\begin{array}{l}\text { CT, CTA, DSA, 3D reconstruction, } \\
\text { CBF/CBV/MTT }\end{array}$ \\
\hline 2 & $38, F$ & 3.3 & Cervical spinal cord chordoma & Endovascular PED & MRA, DSA, MRI, 3D reconstruction \\
\hline 3 & $76, \mathrm{M}$ & 8 & Microcystic adnexal carcinoma & ICA stent \& angioplasty & DSA, MRI \\
\hline 4 & $40, M$ & 36 & Craniopharyngioma & Multiple resections, radiation \& chemo & CT, CT perfusion, MRI, DSA \\
\hline
\end{tabular}

$\mathrm{CBF}=$ cerebral blood flow; $\mathrm{CBV}=$ cerebral blood volume; chemo = chemotherapy; $\mathrm{CTA}=\mathrm{CT}$ angiography; $\mathrm{FU}=$ follow-up; $\mathrm{MTT}=$ mean transit time .

patient management decision. Use of antiplatelet therapy (e.g., aspirin), antilipid therapy (e.g., statins), and blood pressure control may be reasonable approaches for the prevention of disease progression. Planning for individualized treatment of radiation vasculopathy involves assessment of patient history with attention to neurological and cognitive deficits (e.g., optic, sensorineural hearing, pituitary endocrinopathy), type of cancer, radiation treatment dose and duration, and associated chemotherapy; evaluation of imaging to document vascular flow, at-risk cerebral penumbra, and secondary lesions (e.g., aneurysms, cavernous malformations, carotid stenosis); optimization of stroke management (e.g., stroke risk factors, anticoagulation, antilipids, diabetes); and consideration of CEA and CAS, depending on vessel anatomy, surgical risk factors, and durability of treatment.

Treatment of intracranial radiation-induced aneurysms by both open and endovascular methods has been described. ${ }^{17,21}$ By 2013, a total of 46 patients and 69 intracranial aneurysms had been reported in the literature, with an average latency of 12 years from the time of radiation exposure to aneurysm presentation for treatment. ${ }^{21}$ Of these aneurysms, $83 \%$ were saccular, 9\% were fusiform, and 9\% were pseudoaneurysms. Interestingly, the median time to presentation was 20 years after brachytherapy, 8 years after focused radiation therapy, 9 years after whole-brain radiation therapy, and 6 years after stereotactic radiosurgery. These aneurysms had a high risk of rupture, although this may have been the result of a selection bias. Nevertheless, these reports indicate that treatment with either traditional open or endovascular methods could be performed successfully.

Comparison of CAS and CEA has been challenging because of the different strategies for these two procedures. A comprehensive meta-analysis by Fokkema et al..$^{10}$ recently evaluated 27 studies including 533 patients with radiation vasculopathy who underwent treatment (361 CASs, 172 CEAs). The perioperative risk for cerebrovascular adverse events was similar in CAS and CEA (3.9\% vs $3.5 \%, p=0.77$ ). Cranial nerve injury was seen in $9.2 \%$ of CEA cases and no CAS cases. Vessel stenosis of $>50 \%$ was higher after CAS than after CEA ( $p<0.003)$. Mortality rates ranged from $0 \%$ to $33.0 \%$ for CAS patients and $0 \%$ to $44.4 \%$ for CEA patients; these rates were highly dependent on the oncological history of the included patients and length of follow-up rather than the surgical treatment. These results suggest that CEA and CAS can both be options for treatment of radiation vasculopathy with good short-term outcomes and similar perioperative complication rates but that CEA offers more potentially durable vessel patency with a higher rate of cranial nerve injury. With the high oncological mortality in this patient population, the durability and low morbidity of either procedure can be useful for clinical decision-making.

CAS has been advocated as a less invasive treatment strategy for postradiation vasculitis, but it has been controversial because of the potential for later in-stent stenosis. An early study by Protack et al. ${ }^{23}$ compared patients who underwent CAS for radiation vasculopathy with patients treated for atherosclerotic disease alone and demonstrated equivalent 30-day mortality and 3-year survival rates for the two groups. However, at 3 years, the stenosis rate $(80 \%$ vs $26 \%, \mathrm{p}<0.05)$ and graft occlusion rate $(21 \%$ vs $0 \%, \mathrm{p}$ $<0.05$ ) were higher for the radiation treatment group. A study by Sadek et al. ${ }^{24}$ showed no difference in 30-day composite outcome (stroke, myocardial infarction, mortality), 50\% vessel stenosis, or $70 \%$ vessel stenosis at 12 months between patients who underwent CAS after radiotherapy and those who did not have radiotherapy. This study also showed no difference in microembolic protection devices used between atherosclerotic and radiation vasculopathy groups, suggesting that there is no difference in direct plaque friability.

\section{Teaching Case Examples}

After institutional board review approval was granted, a retrospective chart review of cases of radiation vasculopathy was performed. Clinical information, imaging, and treatment course were reviewed (Table 1).

\section{Case 1}

A 37-year-old man presented with a history of right frontal astrocytoma diagnosed at 12 years of age that had been treated with resection and whole-brain radiotherapy. He had a noted history of medically refractory epilepsy treated with oxcarbazepine, divalproex sodium, levetiracetam, and a vagus nerve stimulator. After a recent seizure episode and traumatic brain injury, the patient underwent follow-up CT and CT angiography of the head, which demonstrated a 5-mm anterior communicating artery (ACoA) aneurysm with partial thrombosis, calcification originally measuring $10.3 \mathrm{~mm}$, and a known rightsided stroke (Fig. 1A). The patient was awake and conversant but had left hemiparesis and incoordination. DSA showed significant radiation-induced vasculitis around 

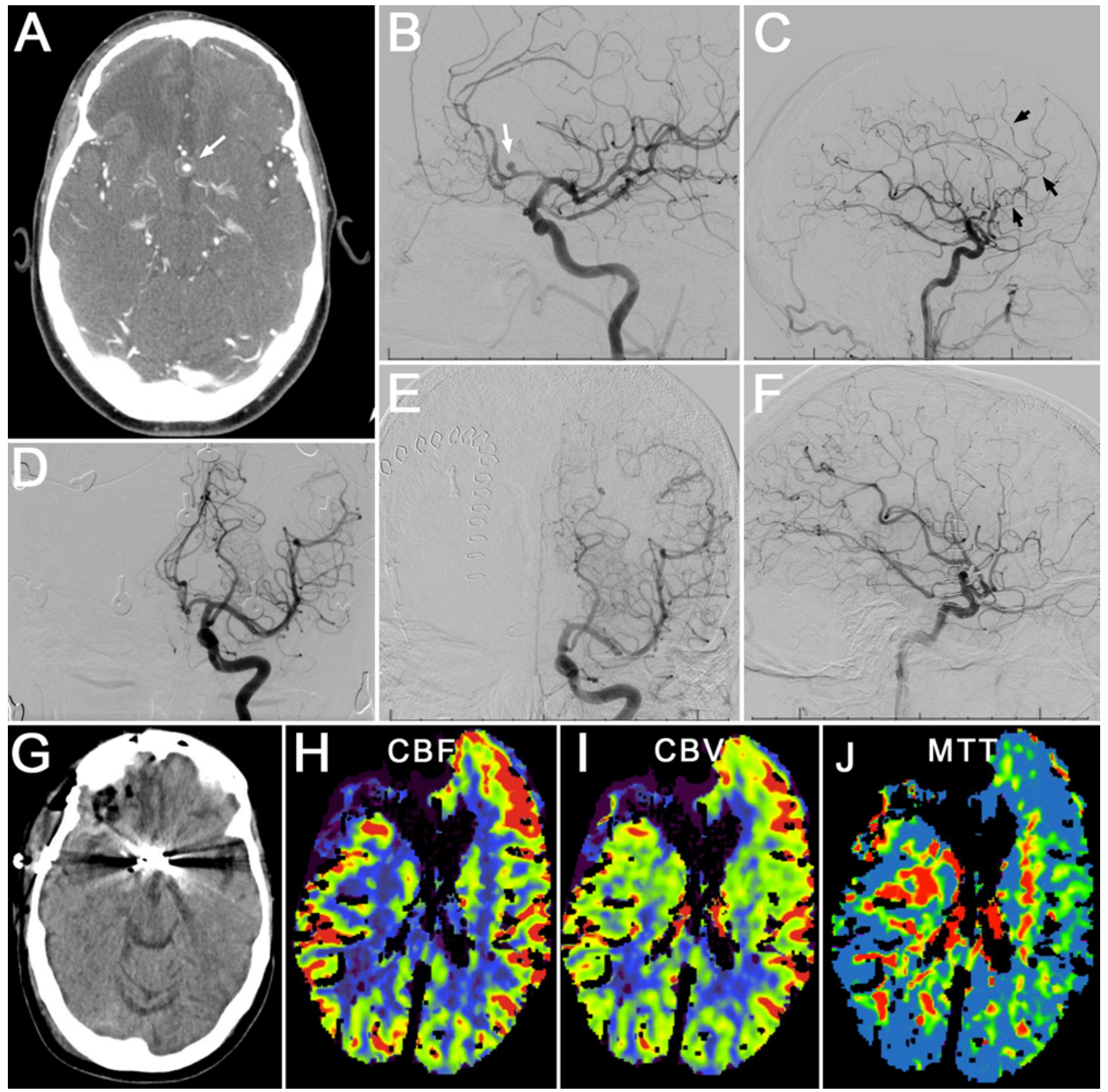

FIG. 1. Case 1. A 37-year-old man presented with newly discovered ACoA aneurysm and a history of radiation vasculitis secondary to treatment of a right frontal astrocytoma at age 12. A: Preoperative CT angiogram demonstrating a 10.3-mm partially thrombosed ACoA aneurysm (arrow). B: Lateral DSA study showing the aneurysm (arrow). C: Lateral DSA study demonstrating radiation vasculitis with decreased arborization and multiple areas of vascular narrowing (arrows). D: Anteroposterior DSA study showing that there were no aneurysmal or vasculitic changes 2 years earlier during a Wada test. E and F: Postoperative anteroposterior (E) and lateral (F) DSA studies showing no residual aneurysm filling after clipping. G-J: CT scan (G) and cerebral blood flow $(\mathrm{CBF}$ ) perfusion $(\mathrm{H})$, cerebral blood volume (CBV) perfusion $(\mathrm{I})$, and mean transit time (MTT) perfusion (J) maps showing no complication. Figure is available in color online only.

the proximal $\mathrm{A}_{1-2}$ complex and the ACoA aneurysm (Fig. 1B-D). Angiography also demonstrated radiation-induced vasculitis. The patient underwent a right frontal pterional craniotomy and aneurysm trapping, which was uneventful. Postoperative DSA showed effective aneurysm occlusion without complication (Fig. 1E-G). A postoperative cerebral perfusion study showed an area of prior infarc- tion known preoperatively (Fig. $1 \mathrm{H}-\mathrm{J}$ ). The patient was monitored during the 23.9-month follow-up.

Because of the patient's specific history of radiation vasculopathy, we decided to treat the aneurysm operatively. Open clipping was thought to be the best option for aneurysm treatment given the patient's vascular fragility. Although endovascular treatment was considered, open 

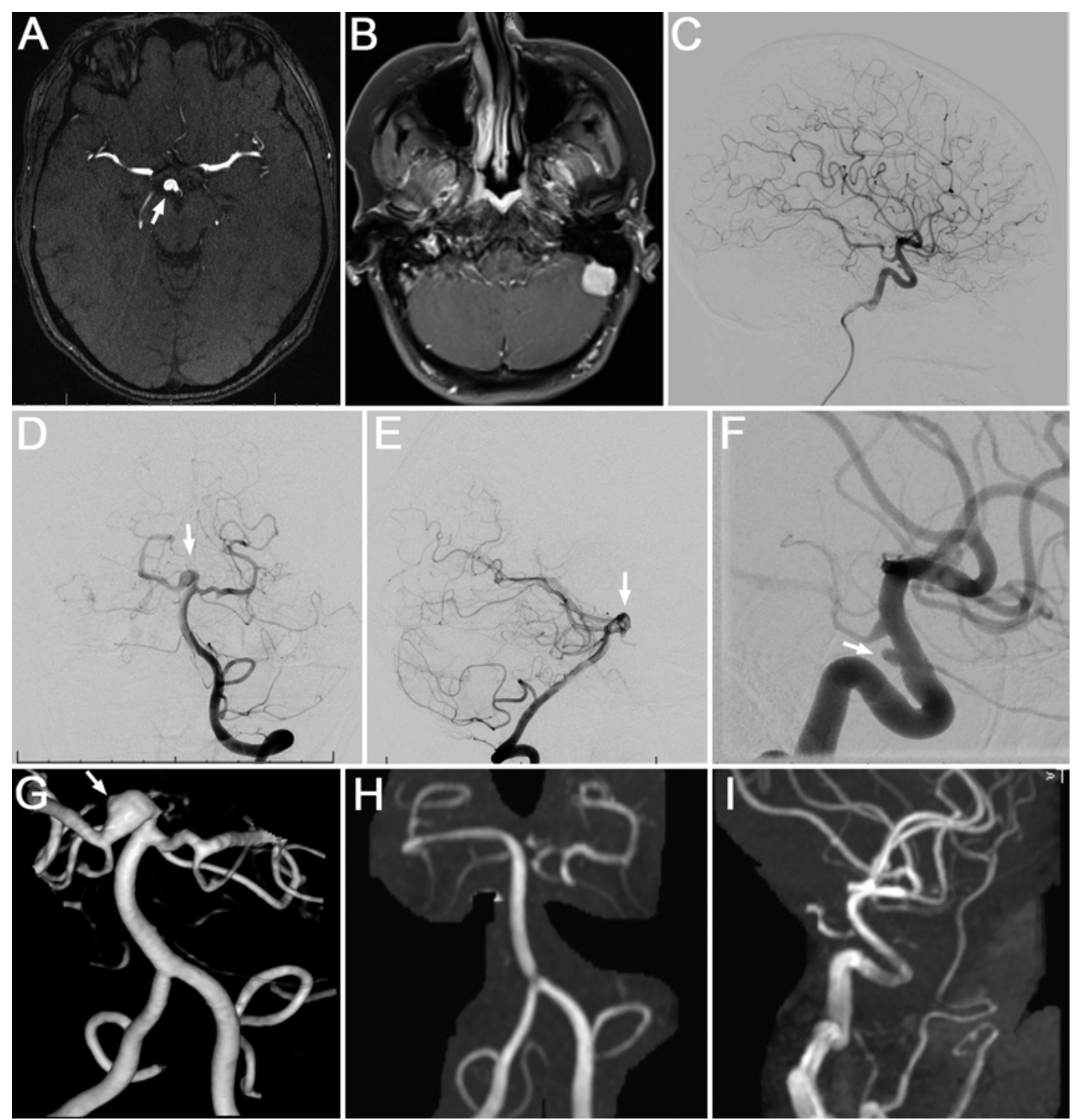

FIG. 2. Case 2. A 38-year-old woman presented with a right $P_{1}$ and left supraclinoid aneurysm after previous treatment of a cervical chordoma with surgery and radiation therapy. A: Preoperative MR angiogram demonstrating a right $P_{1}$ aneurysm (arrow). B: Preoperative MR image showing a known left cerebellopontine angle meningioma also due to prior radiotherapy. C: Lateral DS angiogram showing radiation vasculitis. D and E: Preoperative anteroposterior (D) and lateral (E) DS angiograms showing the posterior communicating artery aneurysm (arrows). F: Lateral left ICA injection showing a superior hypophyseal aneurysm (arrow). G: Three-dimensional reconstruction showing the right $\mathrm{P}_{1}$ aneurysm pointing superolaterally (arrow). $\mathrm{H}$ and I: Follow-up anteroposterior $(\mathrm{H})$ and oblique (I) MR angiograms showing a patent right $\mathrm{P}_{2}$ segment and left ICA.

vascular treatment was thought to offer long-term therapy, with a lower chance of aneurysm regrowth. Evidence in support of open versus endovascular treatments specifically for radiation-induced aneurysms is limited. Generally, the data from the reports of treatment of spontaneous aneurysms have suggested improved safety with endovascular treatment compared with open methods, depending on aneurysm size and location. ${ }^{18}$ However, the durability of endovascular treatments for radiation-induced aneurysms remains to be explored. This patient, who was a long-term cancer survivor and young at the time of presentation, was predicted to show a good life expectancy, thus warranting definitive aneurysm treatment.

\section{Case 2}

A 38-year-old woman presented after routine followup imaging of a known posterior fossa meningioma, neck schwannoma, and thyroid mass. She had a known history of a cervical spinal cord chordoma treated with resection and radiotherapy as well as a diagnosis of a SMARCB1 mutation. In the evaluation of sigmoid sinus patency during workup of the posterior fossa meningioma, MRA demonstrated an aneurysm in the right $\mathrm{P}_{1}$ segment (Fig. 2A and B) and radiation-induced vasculitis (Fig. 2C). Neurologically, the patient exhibited no deficits. She underwent DSA to explore the $\mathrm{P}_{1}$ segment aneurysm (Fig. 2D and E). An incidentally discovered left superior hypophyseal aneurysm 
was identified at this time (Fig. 2F). The patient underwent placement of a Pipeline embolization device (PED) in the right $P_{1}$ segment, which was unremarkable (Fig. 2G). Two months later, she underwent placement of a PED in the left internal carotid artery (ICA), which was again unremarkable. The patient has subsequently been monitored for 3.3 months. Follow-up MRA showed a patent right $\mathrm{P}_{2}$ and left ICA (Fig. 2H and I).

For this patient, identification of dual aneurysms at the $\mathrm{P}_{1}$ and superior hypophyseal segments warranted a strategy to treat simultaneous lesions. Use of the PED, originally aimed at treating the superior hypophyseal aneurysm, allowed effective treatment of both lesions. Antiplatelet therapy with PED placement was thought to be a relative contraindication toward treating one aneurysm openly and another endovascularly. Despite the lack of literature on the use of PEDs in patients with vasculitis, use of the PED here suggested that it might be an effective strategy for the treatment of radiation-induced aneurysms. Because of the more recent development of the PED, data regarding its efficacy in radiation-induced aneurysms are limited to case reports and small case series. Further investigation is required to evaluate whether vascular remodeling, necessary for PED treatment, can occur in a radiation-treated brain.

\section{Case 3}

A 76-year-old man presented with a 1-day onset of recurrent stroke, including symptoms of incoordination. The patient's prior history included stroke ( 7 and 4 years prior), previous proton-beam radiotherapy to his left temporomandibular joint because of a history of microcystic adnexal carcinoma (6 years prior), 4 cerebrovascular stents, and a coronary artery bypass graft ( 7 years prior). New-onset left medullary and left frontal cortex strokes were identified on MRI. The patient underwent DSA, which showed chronic left vertebral artery occlusion, delayed left ICA filling with string sign, and a restenosis proximal to a previously placed carotid stent (Fig. 3A-D). Radiation vasculitis was noted on the patient's angiography examination (Fig. 3E). He exhibited a left superior quadrantanopia and mild gait imbalance on physical examination. He underwent placement of a stent in the left ICA and angioplasty (Fig. 3F), but presented again to the emergency department within 5 days for congestive heart failure exacerbation that was treated with diuresis. Four months later, the patient underwent follow-up angiography that showed restenosis of his left ICA (Fig. 3G and H), requiring angioplasty (Fig. 3I). $\mathrm{He}$ transferred his care 4 months later because of a move.

This case presented a complex situation involving a patient with significant comorbidities and an unclear oncological prognosis. Re-treatment was required because of the higher risk of in-stent restenosis in radiation vasculopathy compared with atherosclerotic vasculopathy. Endovascular treatment of radiation-induced ICA stenosis and vasculitis was selected because of this patient's cardiac risk. Although CAS and CEA are equivalent in terms of perioperative safety, long-term stenosis and mortality vary depending on patient-specific factors. The durability of endovascular treatment is questionable, but one advantage over open surgery may be the potential for repeated treatment. Conclusive evidence regarding the superiority of CAS or CEA for specific patient indications remains to be fully established.

\section{Case 4}

A 40-year-old man presented after multiple previous resections and radiotherapy treatments for a craniopharyngioma that had been diagnosed when the patient was 6 years old (Fig. 4). At age 34 years, the patient had undergone treatment of a radiation-induced aneurysm with stent-assisted coiling. At age 37 years, he underwent a left frontotemporal craniotomy for resection of recurrent craniopharyngioma, and he underwent a second resection along with decompression of the right optic canal because of worsened vision at age 38 years. His most recent symptoms included worsening V1 and V2 distribution numbness, neuralgia, worsened cognition, and progressive growth of the tumor; there was also concern regarding an aneurysm in the cavernous segment of the ICA. The patient underwent a third repeat left frontotemporal craniotomy for resection of recurrent tumor, ligation of cranial nerves III-VI, and exploration of the ICA, which showed tumor invasion but no aneurysm. The patient made an initial uneventful recovery but required eventual placement of a ventriculoperitoneal shunt for the treatment of postoperative hydrocephalus.

This patient's complicated history demonstrates the sequelae of radiation therapy long after completion of treatment. Deficits in vision, endocrine function, and cognitive function all occurred in response to the patient's prior surgical and radiation treatment, which were managed by our neurosurgical team. The patient's most recent surgery presented a dilemma in regard to goals of surgery (e.g., treatment of the aneurysm alone, decompression of cranial nerves, removal of recurrent tumor) and timing of surgery. The high risk of a radiation-induced aneurysm prompted earlier treatment. As after tumor recurrence, the history of radiation vasculopathy and tenuous blood vessels led to operative decompression rather than an attempt at a fourth resection. The previous history of radiation vasculopathy complicated all treatment approaches. These clinical aspects were also critical in the decisions made at various time points during this patient's care to treat a radiationinduced aneurysm and in recommending the patient for reresection.

\section{Case Series Discussion}

Treatment of radiation vasculopathy requires an individualized approach (Table 1). Several commonalities among our cases include the early treatment with radiotherapy to the head or neck for oncological issues with delay in radiation vasculopathy development and the heterogeneity of delayed presentation. Vascular lesions were often identified incidentally after trauma or during seizure evaluation or tumor follow-up. A combination of CT, CT angiography, MR, MRA, MR perfusion, and DSA studies was performed to assess cases. New vascular pathology can develop over a very short time interval, as shown in case 1 , where an angiogram 2 years earlier for a Wada test showed no vascular changes at all, but a follow-up $\mathrm{CT}$ angiogram and then a formal angiogram showed the 
S. Twitchell et al.
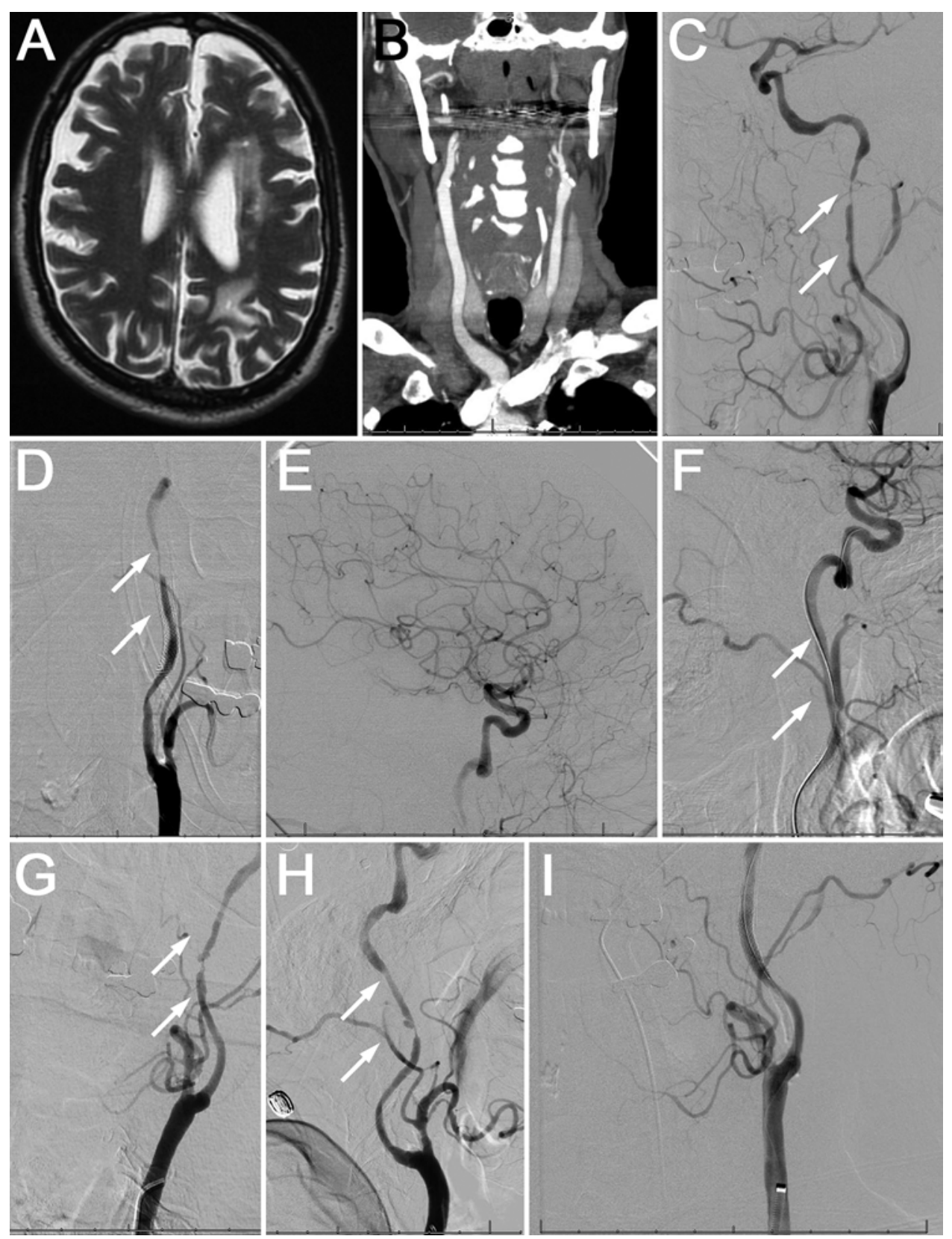

FIG. 3. Case 3. A 76-year-old man presented with a 1-day history of recurrent ischemic stroke and was found to have significant left ICA stenosis and vascular disease due to a prior history of left neck proton-beam radiotherapy for temporomandibular joint microcystic adnexal carcinoma. A: Baseline MR image demonstrating significant vascular disease. B: Previous CT angiogram obtained 7 years prior to presentation, showing adequate neck vessel flow. C and D: Preoperative anteroposterior (C) and lateral (D) images demonstrating significant left ICA stenosis proximal to a prior carotid stent (arrows). Of note, the left vertebral artery also showed chronic occlusion. E: Lateral DS angiogram showing radiation vasculitis. F: Postangioplasty, the stenosis showed significant improvement (arrows). G and H: Anteroposterior $(\mathrm{G})$ and lateral $(\mathrm{H}) \mathrm{DS}$ angiograms obtained 4 months later, showing restenosis at the left ICA (arrows). I: After a second angioplasty, the left ICA shows improved flow.

development of a new anterior cerebral artery aneurysm and severe vasculopathic changes affecting the entire hemisphere. In general, treatment options for secondary radiation-induced aneurysms include both microsurgical clipping and endovascular treatment. Interestingly, treatment of secondary aneurysms with modern flow diverters has the advantage of an endoluminal reconstruction and remodeling of the vessel wall, as seen in case 2. Evidence regarding the selection of treatment options for intracranial radiation-induced aneurysms is lacking and requires further investigation. Treatment of carotid stenosis after radiation therapy can be challenging, as in case 3. CEA is often not the first choice, because of concerns for a "hostile neck" in the presence of the radiation-induced tissue response and the radiation-induced stenosis seen in case 3, which is of a different nature from that of atherosclerotic 

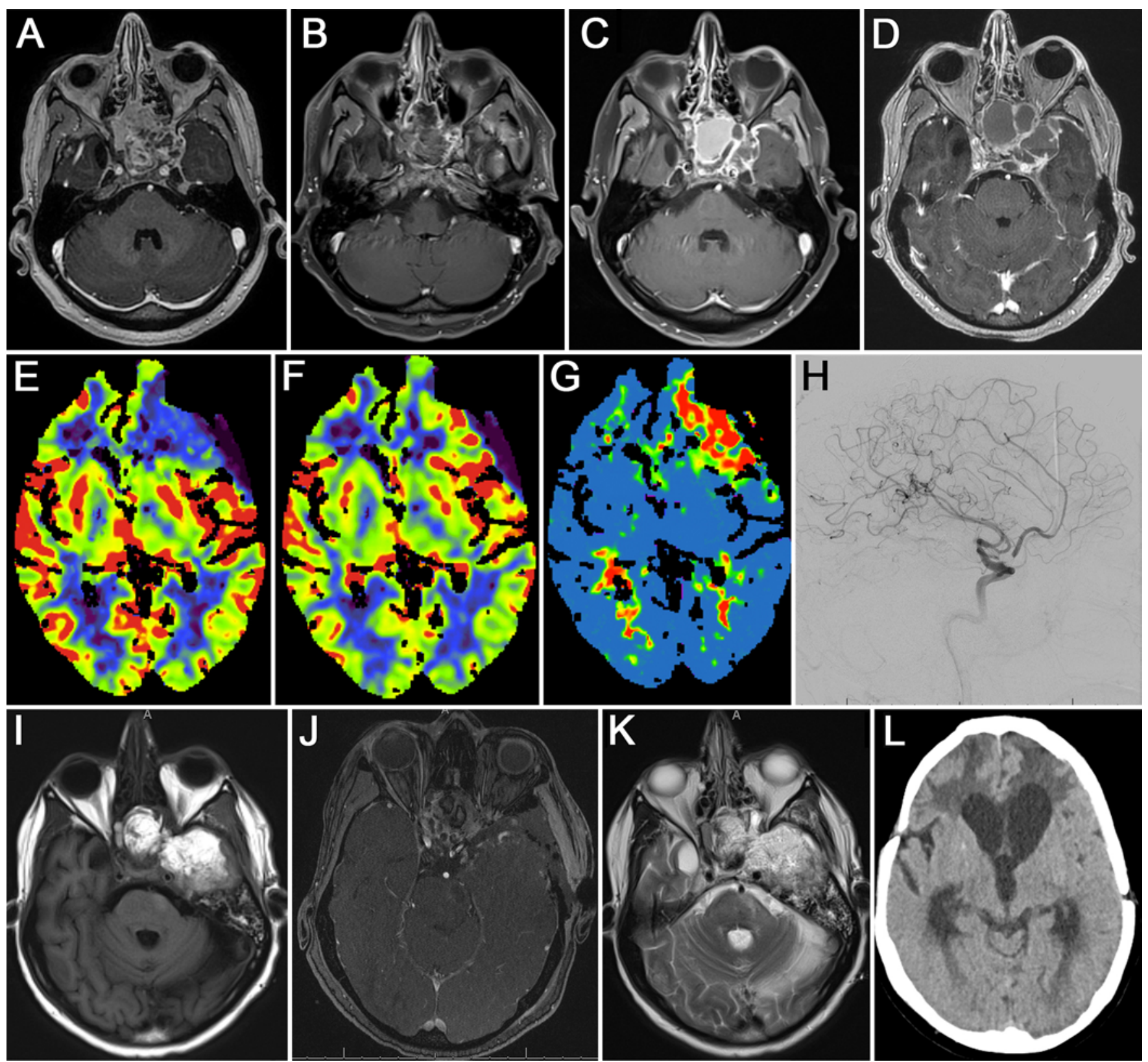

FIG. 4. Case 4. A 40-year-old man presented with a complicated history of recurrent craniopharyngioma after multiple resections, radiation treatment, and chemotherapy beginning at age 6 years. A: Preoperative T1-weighted MR image with contrast showing a multicystic, sellar lesion with surrounding mass effect, which was consistent with recurrent craniopharyngioma. B: Postoperative T1-weighted image with contrast. C: Preoperative T1-weighted image with contrast showing the recurrent lesion with worsened cystic spaces prior to his second resection. D: Preoperative T1-weighted image with contrast, showing recurrence with optic nerve compression prior to his third resection. E-G: Cerebral blood flow perfusion (E), cerebral blood volume perfusion (F), and mean transit time perfusion $(G)$ images obtained after the third resection because of concerns of radiation vasculopathy. H: Lateral diagnostic angiogram showing a prior coiled cavernous segment aneurysm with marked radiation vasculitis within the lateral distribution. I-K: Postoperative T2-weighted (I), T1-weighted with contrast (J), and T1-weighted without contrast (K) MR images demonstrating fat packing around the resection site. L: Follow-up CT scan obtained 3 months later, demonstrating delayed hydrocephalus, which was successfully treated with a ventriculoperitoneal shunt. Figure is available in color online only.

plaque. However, the durability and effectiveness of an endoluminal reconstruction of the vessel after carotid stenting may be limited by the radiation-induced pathology, and, as seen in case 3, multiple stents may be necessary after recurrences. Lastly, case 4 demonstrates the sequelae of radiation vasculopathy, which can occur in a delayed manner and be complicated by tumor recurrence as well as by a patient's other neurological and endocrinological deficits.

One limitation of our series was the small number of cases with heterogeneity of radiation treatment, subsequent clinical management, and follow-up. Multiple treat- 
ment strategies will likely be necessary for the treatment of these patients. Medical management and screening of these patients will surely need standardization as more is understood regarding radiation vasculopathy.

\section{Conclusions}

Radiation vasculopathy remains an underrecognized and poorly managed condition. Pathological features are complex, showing distinct effects at different vessel sizes, and seem to mimic accelerated atherosclerosis. Complications of vasculopathy are heterogeneous and can occur in a delayed fashion from the initial radiation treatment. Advances in medical and surgical management for atherosclerotic disease can be applied to radiation vasculopathy but require further study. Standardized treatment, screening, and guidelines are also necessary for this disease.

\section{Acknowledgments}

We thank Kristin Kraus, MSc, for her editorial assistance.

\section{References}

1. Balentova S, Adamkov M: Molecular, cellular and functional effects of radiation-induced brain injury: a review. Int J Mol Sci 16:27796-27815, 2015

2. Bashar K, Healy D, Clarke-Moloney M, Burke P, Kavanagh E, Walsh SR: Effects of neck radiation therapy on extra-cranial carotid arteries atherosclerosis disease prevalence: systematic review and a meta-analysis. PLoS One 9:e110389, 2014

3. Bhandare N, Antonelli PJ, Morris CG, Malayapa RS, Mendenhall WM: Ototoxicity after radiotherapy for head and neck tumors. Int J Radiat Oncol Biol Phys 67:469-479, 2007

4. Brada M, Burchell L, Ashley S, Traish D: The incidence of cerebrovascular accidents in patients with pituitary adenoma. Int J Radiat Oncol Biol Phys 45:693-698, 1999

5. Brown PD, Foote RL, McLaughlin MP, Halyard MY, Ballman KV, Collie AC, et al: A historical prospective cohort study of carotid artery stenosis after radiotherapy for head and neck malignancies. Int J Radiat Oncol Biol Phys 63:1361-1367, 2005

6. Dearborn JL, Urrutia VC, Zeiler SR: Stroke and cancer-a complicated relationship. J Neurol Transl Neurosci 2:1039, 2014

7. Dietrich J, Gondi V, Mehta M: Delayed complications of cranial irradiation. UpToDate. (https://www.uptodate.com/ contents/delayed-complications-of-cranial-irradiation) [Accessed February 8, 2018]

8. Fajardo LF: Is the pathology of radiation injury different in small vs large blood vessels? Cardiovasc Radiat Med 1:108-110, 1999

9. Flickinger JC, Nelson PB, Taylor FH, Robinson A: Incidence of cerebral infarction after radiotherapy for pituitary adenoma. Cancer 63:2404-2408, 1989

10. Fokkema M, den Hartog AG, Bots ML, van der Tweel I, Moll FL, de Borst GJ: Stenting versus surgery in patients with carotid stenosis after previous cervical radiation therapy: systematic review and meta-analysis. Stroke 43:793-801, 2012

11. Gassmann A: Zur histologie der rontgenulcera. Fortschr Geb Rontgenstrahlen Nuklearmed Erganzungsbd 2:199207, 1899

12. Greene-Schloesser D, Robbins ME, Peiffer AM, Shaw EG, Wheeler KT, Chan MD: Radiation-induced brain injury: a review. Front Oncol 2:73, 2012

13. Haddy N, Mousannif A, Tukenova M, Guibout C, Grill J,
Dhermain F, et al: Relationship between the brain radiation dose for the treatment of childhood cancer and the risk of longterm cerebrovascular mortality. Brain 134:1362-1372, 2011

14. Harris JR, Levene MB: Visual complications following irradiation for pituitary adenomas and craniopharyngiomas. Radiology 120:167-171, 1976

15. Kerklaan JP, Lycklama á Nijeholt GJ, Wiggenraad RG, Berghuis B, Postma TJ, Taphoorn MJ: SMART syndrome: a late reversible complication after radiation therapy for brain tumours. J Neurol 258:1098-1104, 2011

16. Makale MT, McDonald CR, Hattangadi-Gluth JA, Kesari S: Mechanisms of radiotherapy-associated cognitive disability in patients with brain tumours. Nat Rev Neurol 13:52-64, 2017

17. Matsumoto H, Minami H, Yamaura I, Yoshida Y: Radiationinduced cerebral aneurysm treated with endovascular coil embolization. A case report. Interv Neuroradiol 20:448453, 2014

18. Molyneux AJ, Birks J, Clarke A, Sneade M, Kerr RS: The durability of endovascular coiling versus neurosurgical clipping of ruptured cerebral aneurysms: 18 year follow-up of the UK cohort of the International Subarachnoid Aneurysm Trial (ISAT). Lancet 385:691-697, 2015

19. Murphy ES, Xie H, Merchant TE, Yu JS, Chao ST, Suh JH: Review of cranial radiotherapy-induced vasculopathy. J Neurooncol 122:421-429, 2015

20. Muthukrishnan A, Bajoghli M, Mountz JM: Delayed development of radiation vasculopathy of the brain stem confirmed by F-18 FDG PET in a case of anaplastic astrocytoma. Clin Nucl Med 32:527-531, 2007

21. Nanney AD III, El Tecle NE, El Ahmadieh TY, Daou MR, Bit Ivan EN, Marymont MH, et al: Intracranial aneurysms in previously irradiated fields: literature review and case report. World Neurosurg 81:511-519, 2014

22. Plummer C, Henderson RD, O'Sullivan JD, Read SJ: Ischemic stroke and transient ischemic attack after head and neck radiotherapy: a review. Stroke 42:2410-2418, 2011

23. Protack CD, Bakken AM, Saad WE, Illig KA, Waldman DL, Davies MG: Radiation arteritis: a contraindication to carotid stenting? J Vasc Surg 45:110-117, 2007

24. Sadek M, Cayne NS, Shin HJ, Turnbull IC, Marin ML, Faries PL: Safety and efficacy of carotid angioplasty and stenting for radiation-associated carotid artery stenosis. J Vasc Surg 50:1308-1313, 2009

25. Steele SR, Martin MJ, Mullenix PS, Crawford JV, Cuadrado DS, Andersen CA: Focused high-risk population screening for carotid arterial stenosis after radiation therapy for head and neck cancer. Am J Surg 187:594-598, 2004

26. Tabas I, García-Cardeña G, Owens GK: Recent insights into the cellular biology of atherosclerosis. J Cell Biol 209:13-22, 2015

27. Valk PE, Dillon WP: Radiation injury of the brain. AJNR Am J Neuroradiol 12:45-62, 1991

\section{Disclosures}

Dr. Taussky: consultant for Medtronic.

\section{Author Contributions}

Conception and design: Taussky, Couldwell. Acquisition of data: Karsy. Analysis and interpretation of data: Karsy. Drafting the article: Twitchell, Karsy. Critically revising the article: all authors. Reviewed submitted version of manuscript: all authors. Approved the final version of the manuscript on behalf of all authors: Taussky.

\section{Correspondence}

Philipp Taussky: University of Utah, Salt Lake City, UT. neuropub@hsc.utah.edu. 